\title{
El descubrimiento de la insulina
}

\section{The discovery of insulin}

\author{
Jácome $A^{1}$ \\ Este artículo se tomó de: Jácome A. El descubrimiento de la insulina. En: Pinzón Barco JB, editor. \\ Insulinoterapia: una travesía de principio a fin. Bogotá: Distribuna Editorial Médica; 2020. p. 1-10.
}

\begin{abstract}
${ }^{1}$ Miembro de número, Academia Nacional de Medicina de Colombia. Miembro honorario, Asociación Colombiana de Endocrinología, Diabetes y Metabolismo. Editor emérito, Revista Medicina
\end{abstract}

\section{Resumen}

La insulina fue descubierta en Toronto en el verano de 1921. Los gestores del descubrimiento fueron Banting y Best, quienes permanecieron encerrados en aquellos calurosos meses haciendo experimentos en perros. Tanto estos dos investigadores como McLeod, quien era el profesor y jefe de Fisiología en Toronto, y Collip, quién enderezó las investigaciones hacia un producto más puro, reclamaron también su derecho. Zuelzer, en Alemania, Paulesco, en Rumania, y Scott, en Estados Unidos, lo hicieron igual y con anterioridad. Todos estos fueron rescatados por los historiadores en décadas posteriores, pero el premio Nobel lo ganaron Banting y McLeod, y con ellos perdurará el recuerdo.

Palabras clave: insulina, laboratorios Connaught, Frederick Banting, Charles Best, Universidad de Toronto.

\section{Abstract}

Insulin was discovered in Toronto during summer of 1921. Credit for discovery has traditionally been given to Banting and Best, two researchers that remained enclosed in a laboratory during that hot season, performing experiments in a number of dogs. These two researchers claimed being the ones to make that discovery, but so did McLeod, Professor and Chief of the Department of Physiology at University of Toronto, and Collip, who led research towards obtaining a less contaminated hormonal preparation. The German Zuelzer, the Romanian Paulesco and the American Scott, performed similar experiments with pancreatic extracts and obtained same results, although they did them before the Canadians. Work done by the latter was rescued by historians in later years, but Nobel Prize was won by Banting and McLeod, and the milestone will most likely be linked to them.
Key words: insulin, Connaught laboratories, Frederick Banting, Charles Best, University of Toronto.

\section{Introducción}

Desde finales del siglo XIX, varios investigadores intentaron aislar la insulina a partir de páncreas de mamíferos, pero fueron Frederick Banting y Charles Best los que tuvieron éxito, en el verano de 1921. Esto ocurrió en la Universidad de Toronto gracias a la tenacidad del ortopedista Banting, quien tuvo una idea luminosa para lograr el importante hallazgo científico, con la ayuda de Best, un estudiante de Medicina, que, sin embargo, ya tenía un título de fisiólogo. También influyó la cooperación posterior del profesor y jefe de fisiología de la misma universidad, John James Rickard Macleod, y del bioquímico James B. Collip (1-4). Aunque esta es la forma convencional de ver el asunto, hay historiadores que le dan más crédito a Paulesco $(5,6)$ o a Collip (7).

Ante la pregunta ¿quién descubrió la insulina?, se ha dicho que primero fue una idea que conformó una hipótesis, la que intentó comprobarse; finalmente, fue la convicción de Banting (y también de Paulesco) para lograr la obtención de un preparado útil para tratar diabéticos.

\section{Se descubren los islotes}

Paul Langerhans (1847-1888) -siendo estudiante- descubrió los islotes pancreáticos, que describió como "montoncitos de células" en su tesis de grado. Langerhans fue un médico berlinés, que tuvo como profesores a Haeckel, Conheim y Virchow. Entre el verano de 1867 y el otoño de 1868 realizó investigaciones sobre la estructura del páncreas, tema de su tesis de doctorado. En su experimentación utilizó, sobre todo, conejos a los que inyectaba un colorante (azul de Prusia) en el conducto pancreático para visualizar las ramificaciones y la estructura del sistema excretor. Así descubrió las células glandulares que secretan las enzimas digestivas pancreáticas. Distinguió varios tipos celulares, entre estos unas células pequeñas, poligonales, sin gránulos, que tenían el aspecto de manchas diseminadas en el interior del parénquima. Las 
describió como células pequeñas, de contenido prácticamente homogéneo y de forma poligonal, con núcleo redondeado sin nucleolo y unidas siempre de dos en dos o formando pequeños grupos. El patólogo admitió que ignoraba la función de estas células. En 1893, el francés Gustave-Édouard Laguesse afirmó que quizá fabricaran algún producto de secreción interna y las denominó islotes de Langerhans (8).

\section{Diabetes por pancreatectomía}

El experimento más connotado de cirugía experimental en el estudio de un trastorno metabólico fue el que realizó el cirujano Oscar Minkowski (1858-1931) -con la colaboración del farmaceuta Josef von Mering- en 1889. Este último estaba interesado en los procesos de la digestión de las grasas y en el papel del páncreas, por lo que ligó el conducto de este órgano para observar sus efectos, aunque la ligadura dio efectos parciales. Tal vez basado en una afirmación de Claude Bernard unos años antes, creyó que los animales con los que experimentaba morirían si les hacía una pancreatectomía total, en la cual no era tampoco experto. En un encuentro casual con Minkowski discutió su hipótesis sobre la importancia del desdoblamiento de las grasas en ácidos grasos libres para la emulsión y absorción de una comida rica en grasas. Para ver si existía dicha participación pancreática en la digestión, procedieron a la pancreatectomía de unos perros en el laboratorio de Naunyn, experto en diabetes. Aunque en el momento de la experimentación los dos alemanes desconocían la literatura sobre resultados de pancreatectomías, ambos eran personas interesadas en el estudio del metabolismo de los carbohidratos. Cuando semanas después tuvieron anotadas todas las observaciones, hicieron una corta publicación sobre los resultados de la operación. Los animales mostraban una sed insaciable, una elevada producción de orina, pérdida de peso a pesar de buena ingesta y un gran apetito; curiosamente, estos eran los síntomas de la diabetes. Fue Minkowski -por ausencia de Von Mering- quien detectó la glicosuria en la orina de estos perros, sorprendido ante el hecho de que el animal entrenado orinaba por todo el laboratorio. La glicosuria persistió a pesar de un ayuno de dos días o una dieta hecha solo a base de carnes. Se presentó también cetonuria y el glicógeno desapareció de los tejidos donde habitualmente se encuentra, hígado y músculo. La diabetes experimental les hizo deducir que el páncreas contenía alguna sustancia crucial para el metabolismo de la glucosa, por lo que Von Mering consideró que el páncreas realizaba dos funciones, una externa -la producción del jugo pancreático para la digestión de los alimentos- y otra interna, que producía una sustancia reguladora de la glucemia (algo parecido a las conclusiones de Claude Bernard sobre las funciones del hígado). Minkowski intentó disminuir la glucemia con extractos pancreáticos, pero sin resultados.

Aquellos fueron los tiempos de la guerra franco-prusiana, que según Rachmiel Levine aportó algunas cosas al conoci- miento de la diabetes, una de las cuales fue la mejoría de la enfermedad en los enfermos sometidos a la hambruna que causó el sitio de París; la otra, el hecho de que al quedarse con la región de Alsacia-Lorena, los alemanes invirtieron en el desarrollo de la Universidad de Estrasburgo, donde fue nombrado Bernard Naunyn -el principal internista y diabetólogo europeo- director de Medicina. Entre las personas que se llevó a trabajar estaban, además de los dos investigadores que hemos mencionado, el famoso farmacólogo Schmiedeberg -con quien Naunyn editó una prestigiosa revista- y otros como Ernst Stadelmann y Adolf Magnus-Levy, quienes -con Minkowski- demostraron la acidosis diabética por el ácido beta-hidroxibutírico. Todos estos trabajos contaron con el apoyo y supervisión de Naunyn, así como con la producción de una diabetes experimental floriczínica por Von Mering y luego la producida por pancreatectomía por Mering y Minkowski (8).

\section{Comprobando la hipótesis de la insulina}

Con estos antecedentes, ya se pensaba que en el páncreas debía haber una sustancia u hormona, que Meyer, en 1909, había denominado insulina, en caso de que existiese. Después de los experimentos de Oscar Minkowski, que comprobaron la diabetes experimental, Opie y Sobolev, de manera independiente, habían afirmado que los islotes pancreáticos eran necesarios para el control metabólico de los carbohidratos y que la patología de estas células era la responsable de la diabetes (9).

El internista Georg Ludwig Zuelzer había preparado un extracto pancreático que había administrado en perros con glicosuria causada por epinefrina -e incluso a algunos pacientes- y observó algunos efectos hipoglicemiantes, pero dicho extracto resultó tóxico por ser a base de alcohol. El rumano Nicolae Paulesco había obtenido observaciones parecidas, pero no había podido continuar sus experimentos debido a la Gran Guerra. El fisiólogo Marcel Eugène Gley había depositado una carta sellada en la Sociedad de Biología de París, para abrirla hasta que él lo ordenara. En la carta titulada afirmaba que había preparado un extracto a base de los restos atróficos de páncreas de conducto ligado, que había disminuido la glicosuria de un perro pancreatectomizado. Hay quienes dicen que este francés tenía poca confianza en sus investigaciones. También trabajaron en el tema los norteamericanos Israel Kleiner, Ernest Scott y John Murlin y Benjamin Kramer (10-12).

El caso del fisiólogo Paulesco -de Bucarest- es, al parecer, más injusto. Los rumanos protestaron fuertemente por la adjudicación del Nobel de la insulina a los canadienses, pues consideraban que su compatriota fue el primero que había hecho el descubrimiento. En 1916, él tuvo éxito en el desarrollo de un extracto pancreático acuoso que normalizaba la glicemia en los perros diabéticos, pero no pudo continuar sus experimentos debido a la primera gran conflagración europea. Sin embargo, al finalizar la guerra, continuó con sus experimentos 
y aisló la pancreína, su versión de la insulina. Dicen sus defensores que entre abril y junio de 1921, Paulesco presentó cuatro trabajos ante la sección rumana de la Sociedad de Biología de París (que resumían sus investigaciones) y luego logró publicar en el número de agosto de 1921 de la revista belga Archives Internationelles de Physiologíe un extenso artículo sobre el papel del páncreas en la asimilación de los alimentos. En abril de 1922 consiguió una patente del gobierno rumano para la fabricación de la pancreína, pero estos hechos fueron ignorados por la comunidad internacional. Intentos posteriores de dar crédito a los trabajos del rumano se frenaron por razones políticas, ya que el fisiólogo consideraba que había un complot judeo-masónico contra la nación rumana. En todo caso, los canadienses fueron más eficientes y rápidos; trabajaron duro, en condiciones difíciles $(13,14)$.

\section{Una idea que se volvió obsesiva}

A finales de octubre de 1920, el médico canadiense Frederick Banting (1891-1941) era preparador de fisiología en la escuela de medicina en London, Ontario. Después de servir como combatiente en la Primera Guerra Mundial, regresó para ejercer en su región de origen y completó su tiempo del consultorio dictando clases. Tenía experiencia quirúrgica en ortopedia infantil, ya que había trabajado en el Hospital para Niños Enfermos de Toronto. Sin ser un científico, en el fondo tenía la vocación. Cuando le asignaron dictar una clase sobre fisiología del páncreas, se encontró -de manera casual- el número más reciente de Surgery, Gynecology \& Obstetrics y leyó un artículo de Moses Barron sobre un caso de litiasis pancreática encontrado al azar después de cientos de autopsias (15). En la necropsia, se encontró una atrofia de los ácinos con persistencia de las células de los islotes, algo parecido a lo que se observaba al bloquear por ligadura un conducto pancreático. Barron, nacido en Rusia, pero educado en Minnesota, confirmó de esta manera lo que su compatriota Sovolev había descubierto antes en conejos, perros y gatos, pues al ligar el conducto pancreático empezaban a atrofiarse los ácinos productores de enzimas y quedaba como remanente el tejido correspondiente a los islotes de Langerhans. Es decir, había dos tipos de tejidos pancreáticos con productos y acciones totalmente diferentes (1).

A Banting, las conclusiones del artículo le daban vueltas en la cabeza. Como esa noche no pudo conciliar el sueño, se levantó en la madrugada y escribió: “Diabetes. Ligar el conducto pancreático del perro. Mantener los perros vivos hasta que se degeneren sus ácinos y queden los islotes. Tratar de aislar la secreción interna de estos para aliviar la glicosuria” (1).

\section{Por fin, la insulina}

Banting se propuso aclarar el asunto, pero al no encontrar ayuda en su facultad, le recomendaron que acudiera al profesor
John James Rickard Macleod, jefe de fisiología de la Universidad de Toronto, para exponerle su idea simplista y pedirle ayuda.

Convencer a Macleod no fue fácil, pues él -aunque era conocedor del metabolismo de los carbohidratos- tenía la idea prevalente en Europa de que el control de la glicemia tenía lugar en algún centro del sistema nervioso central. Es de anotar que sería de gran ayuda la tecnología reciente, que permitía medir la glicemia con un volumen de sangre cien veces menor, más exacto y rápido $(16,17)$. Con eso y todo, McLeod exigió que se midiera la relación glicemia/nitrógeno ureico, que se consideraba más representativa del estado metabólico. Un desinformado Banting tenía la idea de que el problema de la diabetes era la glicosuria, parámetro que pensaba medir en sus futuros experimentos.

Este joven inexperto no podía lograr mejores resultados que los fisiólogos y los verdaderos investigadores que fallaron en el intento, pensaba el profesor. En la época, había un axioma que decía: "Lo que el jefe dice siempre es correcto". Pero eso no iba con Banting, que era insistente y en tres visitas seguidas logró su objetivo. En el verano de 1921, Macleod le prestó, con displicencia, su laboratorio y 10 perros, y como un proyecto de verano le asignó como asistente a Charles Best, un futuro estudiante de Medicina que tenía ya un grado de baccalaureate en Fisiología y Bioquímica (1). El otro candidato para investigar era su compañero Edward Clark Noble -quien perdió su opción tras el lanzamiento de una moneda- y fue asignado a un proyecto en otra institución (4). No fue mucho lo que se les dio a estos ilusos, pero así se gestó el descubrimiento de la insulina. En un comienzo, Macleod estuvo allí, y su idea era, guardada las proporciones, la de trasplantar el tejido remanente posligadura, pero pronto viajó a Escocia de vacaciones.

Solos ya -aunque en comunicación con Macleod- los canadienses fracasaron en el intento de atrofiar totalmente el páncreas mediante la ligación del conducto de Wirsung; hubo recanalización, se presentaron infecciones y en poco tiempo murieron 7 de los 10 perros. Sin perder su entusiasmo, salieron a comprar perros callejeros económicos, en un momento en el que había gran activismo entre los protectores de animales. El dinero para esto provino de la venta de un carro marca Ford que Banting tenía. Pronto encontraron que no era necesaria la ligadura del conducto e idearon una técnica para retirar todo el páncreas y buscar la atrofia. Luego lograron hacer un extracto del páncreas remanente; tajadas de este fueron colocadas en solución de Ringer, enfriadas y maceradas en mortero y luego filtradas. Una hora después de inyectada la solución a un terrier diabético en coma, este se levantó y movió la cola. Su glicemia descendió para volver a subir después de pasar azúcar por una sonda nasogástrica, aunque ni la hiperglicemia ni la glicosuria fueron tan marcadas como sucedió con un perro pancreatectomizado, al que no se le dio el extracto $(4,5)$. Los dos investigadores habían hecho un descubrimiento extraordinario (Figura 1). 
Figura 1. Placa sobre el descubrimiento de la insulina, erigida por la Ontario Heritage Foundation, Ministerio de la Cultura y de la Comunicación del Canadá. Tomado de: The discovery of insulin por Cria-cow, que está autorizado bajo la licencia CC BY-NC-SA 2.0. Creative Commons autoriza su reproducción sin fines comerciales.



Banting y Best visitaron mataderos y carnicerías para conseguir páncreas de ganado vacuno. También supieron por la literatura que los páncreas fetales tenían más islotes y menos ácinos y empezaron a usarlos. Asimismo, utilizaron páncreas de cerdo para obtener "isletina" en cantidades pequeñas. Al regreso de Macleod, Banting y Best tenían debidamente documentados los resultados sobre 72 perros hechos diabéticos. A pesar de esto, Macleod ordenó repetir los experimentos, lo que hicieron (18-20). En los experimentos también se había usado la secretina para dejar exhausto el páncreas de su jugo. En opinión de Michael Bliss, el historiador más importante de lo ocurrido en Toronto, "La investigación realizada por Banting y Best fue tan mal hecha, que sin la ayuda posterior de Macleod y de Collip [...] el destino de los 2 jóvenes canadienses hubiera sido el de desaparecer en la historia de la medicina" $(1,2)$. Con todo, Macleod ordenó que la totalidad del esfuerzo del departamento se dedicara a la investigación de la insulina.

A Banting no le caía bien el escocés y sus suspicacias y exigencias le producían más escozor. Estuvo a punto de dejar todo, pero Best - con quien había desarrollado una excelente relación- lo convenció de no hacerlo. En una presentación inicial ante un grupo de fisiólogos (posteriormente publicada), el manejo que le dio Macleod a la presentación hizo pensar que él y su grupo habían descubierto la insulina (21). Al año siguiente, sus investigaciones fueron publicadas en el Canadian Medical Association Journal (22). Fue un informe preliminar, seguido de otro más detallado (23). Más tarde, se sumaría el bioquímico James B. Collip al grupo, para hacer importantes contribuciones en la purificación del extracto insulínico, lo que permitió su uso en una buena cantidad de pacientes que empezaron a llegar ante la dispersión de la noticia, de que una nueva droga podía salvarlos. Eli Lilly \& Co., (Novo) Nordisk, a través de Krohl, y Wellcome colaboraron en la comercialización de la insulina $(1-5,24)$.

\section{Los laboratorios Connaught}

Un médico de la Universidad de Toronto y bacteriólogo del Instituto Pasteur, John G. Fitzgerald, estableció un laboratorio para producir antitoxina diftérica en una finca de caballos, herencia de su mujer. Al trasladarse a una finca más grande, la empresa adoptó el nombre de Connaught Antitoxin Laboratories and University Farm, por el duque de Connaught, que era el gobernador general de Canadá en la época de la Primera Guerra Mundial. Aunque este laboratorio siempre ha estado dedicado a la investigación y producción de vacunas, fue uno de los receptores del descubrimiento de la insulina (por su conexión con la Universidad de Toronto) y Charles Best fue su presidente. Este hecho impulsó el rápido crecimiento y el desarrollo de métodos de producción de la insulina a gran escala. Álvaro Morales Gómez, un urólogo colombiano egresado de la Universidad Javeriana de Bogotá -y compañero de clase de quien esto escribe- hizo allí parte de su entrenamiento, investigó y fue conferencista de esta institución, aunque su fuerte es la andrología y la urología oncológica. De paso hay que anotar que también hizo parte de sus estudios en la Universidad de Aberdeen, Escocia, donde estudio Macleod. Connaught hace parte ahora de Sanofi-Pasteur, la rama de Sanofi-Aventis dedicada a las vacunas.

Macleod consideraba que había dado suficiente crédito a los investigadores originales, tanto, que declinó el ofrecimiento de poner su nombre en la lista de autores del artículo sobre la insulina. La relación entre Banting y Macleod empeoró cuando ambos ganaron el Nobel de Medicina en 1923; el ortopedista entonces compartió su premio con Best; el escocés hizo lo propio con Collip. Banting denigraba a Macleod por un conflicto de personalidades. Los argumentos de Best en cuanto al Nobel se debieron a su sed de reconocimiento.

El preparado de Collip empezó a usarse en pacientes jóvenes gravemente descompensados, entre los que se encontraban Leonard Thompson, de 14 años, Elizabeth Hughes (hija del secretario de estado de los Estados Unidos) y varios enfermos más (25). Macleod luego estuvo extrayendo insulina de ciertos peces ricos en ella, con la idea de ayudar en la producción, pero la idea se desechó (26).

Banting fue nombrado profesor en la universidad, pero al incorporase nuevamente al ejército, perdió la vida en un accidente aéreo en el tiempo de la Segunda Guerra Mundial, a los cuarenta y nueve años. En sus últimos tiempos se había dedicado a estudiar la fisiología pulmonar (18). Macleod fue mé- 
dico de la Universidad de Aberdeen -en su país natal-, luego estudió en Leipzig, fue demostrador de fisiología en la facultad de Medicina de Londres, donde también dictó conferencias de bioquímica, hasta el momento una ciencia en ciernes. Posteriormente, se trasladó a Cleveland (Universidad Case Western Reserve) y finalmente fue elegido profesor de Fisiología en la Universidad de Toronto. Allí se dedicó a trabajar en el metabolismo de los carbohidratos. Escribió once libros (1).

La decisión sobre quién descubrió la insulina fue de la Comisión del Nobel de Medicina, después de la visita de uno de ellos a las instalaciones de Toronto. Sigue siendo una decisión polémica un siglo después, ya que los otros investigadores citados (Zuelzer, Paulesco y Scott) también inyectaron un extracto pancreático con alto grado de impureza, que igualmente redujo los niveles de glucemia, y esto lo hicieron antes que los canadienses (27). No hay duda de que el emprendedor fue Banting, ni de que el fisiólogo Best le ayudó en la investigación, pero sin la repetición de los defectuosos experimentos ordenada y guiada por McLeod, sin las técnicas de purificación que aportó Collip y sin la entrada en escena de la casa americana Lilly para la fabricación industrial, el hecho no habría pasado de ser anecdótico. Existen bastantes argumentos a favor de los otros científicos, pero aparte de las discusiones académicas de los historiadores, el recuerdo continuará ligado a los que ganaron el Premio Nobel.

\section{La era postinsulina}

El gran clínico americano Elliot P. Joslin se dio cuenta, sin embargo, de que solucionar el problema diabético no era así de simple; por supuesto que antes de Banting, Best y Macleod, dos de cada tres diabéticos con cetoacidosis morían y para evitarlo acudían a las dietas emanciantes de Allen; con la insulina, la mortalidad por esta complicación aguda se redujo a su mínima expresión. Al prolongarse la vida del diabético, pasaron entonces las complicaciones crónicas a constituirse en el problema real (1)

Frederick Sanger dilucidó la estructura proteica de la hormona. Más tarde, Steiner descubrió el precursor proinsulina. En la década de los 30, Abel, y luego Scott, lograron cristalizar la hormona y hacer preparaciones puras de esta. Para esta época, en Copenhague, el grupo formado por Hans Christian Hagedorn y Birger N. Jensen, con algunas luces del nobel Khrogh, interesado también en la insulina, presentaron en 1936 una insulina de absorción lenta, que resultó de la unión de la hormona con la protamina, una proteína básica. Con esta molécula se lograron preparados comerciales de insulina, como la protamina zinc y otras. La estructura cristalina de la insulina en estado sólido fue determinada por Dorothy Hodgkin.

Cuenta el endocrinólogo colombiano Efraim Otero que, en 1958, cuando era residente de endocrinología del Presbyterian Hospital, Universidad de Columbia en Nueva York, tuvo la oportunidad de conocer personalmente al señor Colt, uno de los primeros pacientes del profesor Palmer, jefe de medicina interna de Columbia. Este enfermo era un diabético juvenil tratado veinticinco años atrás con los primeros extractos impuros procedentes de Toronto, que le salvaron la vida y le permitieron sobrevivir todo ese tiempo. Los cálculos hechos en ese entonces mostraron que las dosis salvadoras, en términos modernos, probablemente no habrían excedido la cantidad de una a dos unidades internacionales diarias (8).

\section{Referencias}

1. Bliss M. The discovery of insulin. Chicago: The University of Chicago Press; 1982.

2. Karamitsos DT. The story of insulin discovery. Diabetes Res Clin Pract. 2011;93(Suppl 1):S2-8. doi: 10.1016/S0168-8227(11)70007-9

3. Best $\mathrm{CH}$. Reminiscences of the Researches which Led to the Discovery of Insulin. Can Med Assoc J. 1942;47(5):398-400.

4. Rafuse J. Seventy-five years later, insulin remains Canada's major medicalresearch coup. CMAJ.1996;155(9):1306-8.

5. Medvei VC. A History of Endocrinology. Lancaster, Boston: MTP Press Limited; 1982.

6. Paulesco NC. Action de l'extrait pancréatique injecté dans le sang, chez un animal diabétique. C.R. Soc. Biologie. 1921;85:555-559.

7. Lestradet H. [The discovery of insulin]. Bull Acad Natl Med.1996;180(2):437-45.

8. Jácome-Roca A. Historia de las hormonas. Bogotá: Academia Nacional de Medicina. Prismagraf Editores; 2008.

9. Opie EL. The relation of diabetes mellitus to lesions of the pancreas. Hyaline degeneration of the islands of Langerhans. J Exp Med. 1900;5(5):52740. doi: $10.1084 /$ jem.5.5.527

10. Scott EL. On the influence of intravenous injections of an extract of the pancreas on experimental pancreatic diabetes. Am J Physiol. 1912;29(3):30610. doi: 10.1152/ajplegacy.1912.29.3.306

11. Kleiner IS. The action of intravenous injections of pancreas emulsions in experimental diabetes. J Biol Chem. 1919;40:153-70.
12. Murlin JR, Kramer B. The influence of pancreatic and duodenal extracts on the glycosuria and the respiratory metabolism of depancreatized dogs. Proc Soc Exp Biol Med. 1913;15:365-383.

13. Rosenfeld L. Insulin: discovery and controversy. Clin Chem. 2002;48(12):2270 88.

14. Alpert JS. An Amazing Story: The Discovery of Insulin. Amer J Med. 2016;129(3):231-2. doi: 10.1016/j.amjmed.2016.01.001

15. Barron M. The relation of the islets of Langerhans to diabetes with special reference to cases of pancreatic lithiasis. Surg Gynec Obstet. 1920;31:43748.

16. Lewis RC, Benedict SR. A method for the estimation of sugar in small quantities of blood. J Biol Chem. 1915;20:61-72.

17. Folin $\mathrm{O}, \mathrm{Wu}$ H. A system of blood analysis. Supplement I. A simplified and improved method for determination of sugar. J Biol Chem. 1920;41:367 374.

18. Bliss M. Rewriting medical history: Charles Best and the Banting and Best myth. J Hist Med Allied Sci. 1993;48:253-74.

19. Collip JB. Frederick Grant Banting, discoverer of insulin. Sci Monthly 1941;52:473-4.

20. Pratt JH. A reappraisal of researches leading to the discovery of insulin. J Hist Med Allied Sci. 1954;9(3):281-9.

21. Banting FG, Best CH, Collip JB, Campbell WR, Fletcher AA, Macleod JJR, Noble EC. The effect produced on diabetes by extracts of pancreas. Trans Assoc Am Physicians. 1922;37:337-47. 
22. Banting FG, Best CH, Collip JB, Campbell WR, Fletcher AA. Pancreatic extracts in the treatment of diabetes mellitus. Preliminary report. Can Med Assoc J. 1922;12:141-6.

23. Banting FG, Best $\mathrm{CH}$. The internal secretion of the pancreas. J Lab Clin Med. 1922;7(5):251-266.

24. Poulsen JE. The impact of August Krogh on the insulin treatment of diabetes and our present status. Acta Med Scand Suppl. 1975;578:7-14. doi: 10.1111/j.0954-6820.1975.tb06497.x
25. Cooper T, Ainsberg A. Breakthrough - Elizabeth Hughes, the Discovery of Insulin, and the Making of a Medical Miracle. New York: St. Martin's Griffin Publishers; 2010.

26. Macleod JJR. The source of insulin. A study of the effect produced on blood sugar by extracts of the pancreas and principal islets of fishes. J Metab Res. 1922;2:149-72

27. Leiva A, Brugués E, Leiva-Pérez A. The discovery of insulin: Continued controversies after ninety years. Endocr Nutr. 2011;58(9):449-56. doi: 10.1016/j.endonu.2011.10.001 

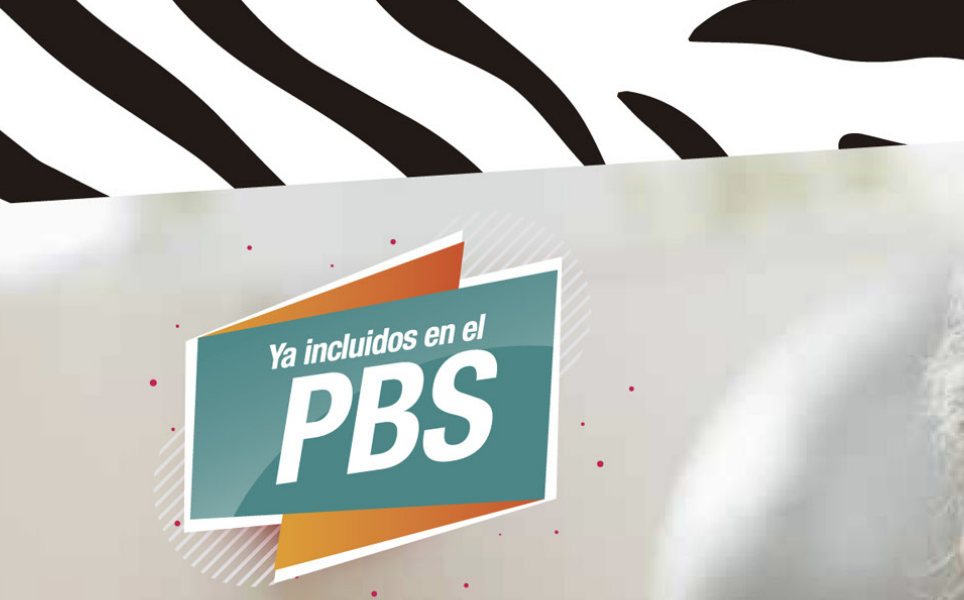

Asegúrese que al hacer el

cambio de una Teriparatida a otra, sea por un medicamento

Eficaz y Seguro.



BIOSETT



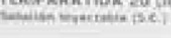

Intercambiar de teriparatida a Biosett ${ }^{\oplus}$

no altera la eficacia ni seguridad del tratamiento para su paciente.



Se comparó el Switch de teriparatida a Biosett $^{\circledR}$ y no se encontraron diferencias significativas en eficacia y seguridad. ${ }^{1}$

Haga el cambio con seguridad a
BIOSIDUS 